\title{
PRE-IMAGES OF EXTREME POINTS OF THE NUMERICAL RANGE, AND APPLICATIONS
}

\author{
ILYA M. SPITKOVSKY AND STEPHAN WEIS
}

\begin{abstract}
We extend the pre-image representation of exposed points of the numerical range of a matrix to all extreme points. With that we characterize extreme points which are multiply generated, having at least two linearly independent pre-images, as the extreme points which are Hausdorff limits of flat boundary portions on numerical ranges of a sequence converging to the given matrix. These studies address the inverse numerical range map and the maximum-entropy inference map which are continuous functions on the numerical range except possibly at certain multiply generated extreme points. This work also allows us to describe closures of subsets of 3-by-3 matrices having the same shape of the numerical range.
\end{abstract}

Mathematics subject classification (2010): 47A12, 54C10, 62F30, 94A17.

Keywords and phrases: Numerical range.

\section{REFERENCES}

[1] Y. H. Au-Yeung, Y. T. Poon, A remark on the convexity and positive definiteness concerning Hermitian matrices, Southeast Asian Bull. Math. 3 (1979) 85-92.

[2] S. K. Berberian, G. H. ORLAnd, On the closure of the numerical range of an operator, Proceedings of the American Mathematical Society 18 (3) (1967) 499-503.

[3] T. Bonnesen, W. Fenchel, Theory of Convex Bodies, B CS Associates, Moscow, Idaho USA, 1987.

[4] E. S. Brown, I. M. SPITKovsky, On flat portions on the boundary of the numerical range, Linear Algebra and its Applications 390 (2004) 75-109.

[5] J. Chen, Z. Ji, C.-K. Li, Y.-T. Poon, Y. Shen, N. Yu, B. Zeng, D. Zhou, Discontinuity of maximum entropy inference and quantum phase transitions, New Journal of Physics 17 (8) (2015) 083019.

[6] M.-T. CHIEN, H. NAKAZATO, Joint numerical range and its generating hypersurface, Linear Algebra and its Applications 432 (1) (2010) 173-179.

[7] D. Corey, C. R. Johnson, R. Kirk, B. Lins, I. Spitkovsky, Continuity properties of vectors realizing points in the classical field of values, Linear and Multilinear Algebra 61 (2013) 1329-1338.

[8] I. CsiszÁr, F. Matúš, Closures of exponential families, The Annals of Probability 33 (2) (2005) $582-600$.

[9] J. EldRed, L. Rodman, I. SPITKovsky, Numerical ranges of companion matrices: flat portions on the boundary, Linear and Multilinear Algebra 60 (2012) 1295-1311.

[10] G. FISCHER, Plane Algebraic Curves, Providence, Rhode Island: AMS, 2001.

[11] T. Gallay, D. Serre, Numerical measure of a complex matrix, Communications on Pure and Applied Mathematics 65 (3) (2012) 287-336.

[12] I. M. Gelfand, M. M. Kapranov, A. V. Zelevinsky, Discriminants, Resultants, and Multidimensional Determinants, Boston, MA: Birkhäuser Boston, 1994.

[13] B. GRÜnbaum, Convex Polytopes, 2nd Edition, New York: Springer, 2003.

[14] P. R. Halmos, Ten problems in Hilbert space, Bull. Amer. Math. Soc. 76 (5) (1970) 887-933.

[15] F. HAusdoRfF, Der Wertvorrat einer Bilinearform, Math. Z. 3 (1) (1919) 314-316.

[16] J. W. Helton, I. M. SPITKOVSKY, The possible shapes of numerical ranges, Operators and Matrices 6 (2012) 607-611. 
[17] R. A. Horn, C. R. Johnson, Topics in Matrix Analysis, 10. printing, Cambridge Univ. Press, 1991.

[18] E. T. JAYNES, Information theory and statistical mechanics, Phys. Rev. 106 (1957), 620-630 and 108 (1957), 171-190.

[19] M. Joswig, B. Straub, On the numerical range map, Journal of the Australian Mathematical Society 65 (1998) 267-283.

[20] K. Kato, F. Furrer, M. Murao, Information-theoretical analysis of topological entanglement entropy and multipartite correlations, Physical Review A 93 (2016), 022317.

[21] D. S. Keeler, L. Rodman, I. M. Spitkovsky, The numerical range of $3 \times 3$ matrices, Linear Algebra and its Applications 252 (1997) 115-139.

[22] R. Kippenhahn, Über den Wertevorrat einer Matrix, Math. Nachr. 6 (1951) 193-228.

[23] T. Leake, B. Lins, I. M. SPITKOVSKy, Pre-images of boundary points of the numerical range, Operators and Matrices 8 (2014) 699-724

[24] T. LEAKE, B. Lins, I. M. SPITKOVSKY, Inverse continuity on the boundary of the numerical range, Linear and Multilinear Algebra 62 (2014) 1335-1345.

[25] C.-K. LI, Y.-T. Poon, Convexity of the joint numerical range, SIAM J. Matrix Anal. A 21 (2) (2000) 668-678.

[26] P. X. Rault, T. Sendova, I. M. Spitkovsky, 3-by-3 matrices with elliptical numerical range revisited, Electronic Journal of Linear Algebra 26 (2013) 158-167.

[27] F. Rellich, Perturbation Theory of Eigenvalue Problems, Research in the Field of Perturbation Theory and Linear Operators, Technical Report No. 1, Courant Institute of Mathematical Sciences, New York University, 1954.

[28] L. Rodman, I. M. Spitkovsky, $3 \times 3$ matrices with a flat portion on the boundary of the numerical range, Linear Algebra and its Applications 397 (2005) 193-207.

[29] L. Rodman, I. M. SPitkovs Ky, A. SzkoŁa, S. Weis, Continuity of the maximum-entropy inference: Convex geometry and numerical ranges approach, Journal of Mathematical Physics 57 (20-16), 015204.

[30] R. SchneIder, Convex Bodies: The Brunn-Minkowski Theory, 2nd Edition, Cambridge University Press, 2014.

[31] O. ToeplitZ, Das algebraische Analogon zu einem Satze von Fejér, Math. Z. 2 (1-2) (1918) 187-197.

[32] S. WEIS, Quantum convex support, Linear Algebra and its Applications 435 (12) (2011) 3168-3188; correction (2012) ibid. 436 xvi.

[33] S. WEIS, A note on touching cones and faces, Journal of Convex Analysis 19 (2012) 323-353.

[34] S. WEIS, Information topologies on non-commutative state spaces, Journal of Convex Analysis 21 (2014) 339-399.

[35] S. WEIS, Continuity of the maximum-entropy inference, Communications in Mathematical Physics 330 (3) (2014) 1263-1292.

[36] S. WeIS, Maximum-entropy inference and inverse continuity of the numerical range, Reports on Mathematical Physics 77 (2) (2016), 251-263.

[37] S. Weis, A. Knauf, Entropy distance: New quantum phenomena, J. Math. Phys. 53 (10) (2012) 102206 